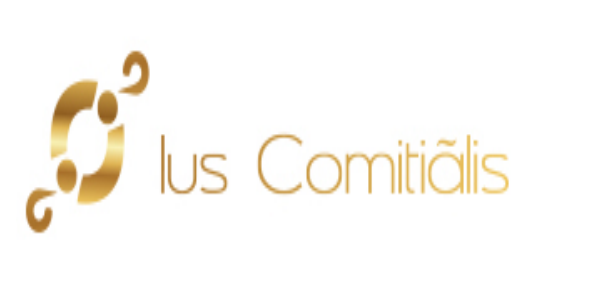

Ius Comitiãlis

ISSN: 2594-1356

iuscomitialis@uaemex.mx

Universidad Autónoma del Estado de México

México

Co\#rdoba Hoyos, Lyda Teresa; Arias Caldero\#n, Jorge Enrique

La democracia participativa como principio constitucional: algunos aportes para la gobernanza del agua en Colombia[1]

Ius Comitiãlis, vol. 2, núm. 3, 2019, Enero-Junio, pp. 238-269

Universidad Autónoma del Estado de México

México

Número completo

Más información del artículo

Página de la revista en redalyc.org 


\section{La democracia participativa como principio cons- titucional: algunos aportes para la gobernanza del agua en Colombia ${ }^{1}$ \\ Participatory democracy as a constitutional principle: Some contributions for the water governance in Colombia}

\section{(c) $(1)(9)$}

Esta obra está bajo licencia Creative Commons Attribution-NonCommercial-ShareAlike $\quad 4.0$ International (CC BY-NC-SA 4.0)

Resumen Este artículo presenta un balance de las perspectivas teóricas y conceptuales con respecto a la democracia representativa y el sentido que ésta tiene cuando se habla de gobernanza. Específicamente, busca exponer una serie de elementos que promuevan la discusión sobre la participación en la gobernanza del agua para lo cual se presentan conceptualizaciones, ya sea explícitas o implícitas sobre el término, así como algunas experiencias concretas de su aplicación y los cuestionamientos que diversos autores han hecho a las definiciones existentes. Se discuten algunos enfoques desde los cuales se puede abordar la participación en sus distintas acepciones y su relación con la gobernanza del agua, todo ello, derivado de una revisión de literatura.

Palabras clave Democracia participativa, Recursos hídricos, Gobierno y gobernanza.

Abstract This article presents a balance of the theoretical and conceptual perspectives with respect to representative democracy and the meaning that it has when talking about governance. Specifically, it seeks to expose a series of elements that promote the discussion on participation in water governance for which conceptualizations are presented, whether explicit or implicit about the term, as well as some concrete experiences of its application and the questions that various authors have made to the existing definitions. Some approaches are discussed from which participation in its different meanings and its relationship with water governance can be addressed, all derived from a literature review.

Key words Participatory democracy, Water resources, Governance and water governance. 


\section{INTRODUCCIÓN}

En Colombia, con la expedición de la Constitución Política de 1991, se dio un vuelco notable al transformar algunas instituciones, particularmente la que toca con la democracia, al pasar de una democracia representativa a una democracia participativa y pluralista, tal como lo consagra el artículo $1^{\circ}$ titulado "De los principios fundamentales", con lo cual eleva la categoría de la democracia participativa y pluralista a un rango de principio fundamental. Esto, a la luz de la interpretación de la Corte Constitucional implica "(...) prescripciones jurídicas generales que suponen una delimitación política y axiológica reconocida y, en consecuencia, restringen el espacio de interpretación, lo cual hace de ellos normas de aplicación inmediata, tanto por el legislador como por el juez constitucional" (Sentencia \# T 406/92, Magistrado Ponente: Ciro Angarita Barón, p. 12) (Sentencia, 1992).

La anterior interpretación de los principios fundamentales consignados en la Carta Política, reviste una gran importancia al momento de la aplicación de los mismos por su eficacia al considerar la Corte Constitucional que cuentan con una mayor capacidad para ser aplicados de manera directa e inmediata, esto es, mediante una subsunción silogística (Sentencia, 1992). Sin embargo, aunque se cuenta con todos estos mecanismos, en materia de derechos ambientales no siempre se aplica la misma lógica, por lo cual es preciso revisar algunos antecedentes en el contexto nacional e internacional con respecto a la participación, la gobernanza y la gestión del agua, y recuperar de ellos los elementos más importantes, que contribuyan, en un futuro cercano a una mejor comprensión del sentido que tiene la democracia representativa en el contexto latinoamericano.

\section{REFLEXIÓN TEÓRICA ACERCA DE LA DEMOCRACIA PARTICIPATIVA}

Si bien, es prácticamente imposible prescindir de la democracia representativa en los Estados modernos de corte liberal, más cuando se hace necesario la implementación de un modelo representativo para los órganos supranacionales, cuya importancia es innegable en un mundo global, no significa que la democracia representativa no sea objeto de serias críticas por diversos sectores de la sociedad para hacer valer una democracia que reconozca la universalidad de los sujetos. Held (1997) afirma: 
Incluso los críticos contemporáneos de las democracias modernas tienden a compartir este supuesto; siguiendo la historia de la democracia tal como habitualmente se relata, han concebido al problema de la accountability política como, ante todo, un problema nacional. Sostienen que las estructuras representativas no son lo suficientemente responsables ante sus ciudadanos; y, al discutir las distintas formas de la democracia directa, o al reivindicar la permanente relevancia del republicanismo, ponen el acento en la necesidad de lograr que el proceso político sea más trasparente e inteligente, más abierto y receptivo a los heterogéneos deseos y necesidades del pueblo.

Entre las críticas al modelo de la democracia representativa están las siguientes:

- Ausencia de espacios y recursos para la deliberación por parte de los ciudadanos.

- Manipulación electoral e ideas falsas sobre la libre elección.

- Ciudadanía excluida o sin libertades reales y efectivas para ejercer sin presiones el derecho al voto consciente.

- Corrupción en las diferentes esferas del sector público que desdibujan las votaciones.

- Poca credibilidad en el sistema democrático y del Estado por parte de la ciudadanía y deslegitimación de la democracia.

- Es un modelo elitista en tanto que un aspirante a un cargo representativo del Estado debe invertir grandes sumas de dinero o hacer parte de la élite del grupo o partido político que lo avala, es decir, no todos tienen igualdad de condiciones para aspirar a ejercer su derecho político de ser elegido.

- Ausencia de responsabilidad jurídica frente al incumplimiento de las promesas realizadas para ser elegido.

- En gran parte de los teóricos se encuentra una postura pesimista de las capacidades del ciudadano para enfrentar debates complejos.

- El problema vedado de la falta de consentimiento en el ejercicio del derecho al voto que invalidaría el sufragio como acto jurídico vinculante.

Frente a la crisis de la democracia representativa los teóricos de los años 60 lanzan la llamada democracia participativa, la cual según viene a corresponder a una subespecie entre democracia electoral y representativa por un lado, y democracia directa y refrendaria por el otro (Sartori, 1994). De igual manera, aclara Sartori que "la democracia representativa no excluye la participación ni el referéndum; más bien los incluye, pero como categorías subordinadas, y de esta manera al no tener una respuesta precisa sobre la democracia participativa, se queda con la duda de si ha nacido algo nuevo o corresponde a una subespecie de democracia directa o de democracia refrendaria, después de haber realizado un análisis pesimista de estas subespecies" (1994, p. 78).

Para los años 60, los teóricos influenciados por el socialismo y los diversos tipos de movimientos sociales de corte ecologista, pacifistas, de género, etc. eran consecuentes con una democracia de asambleísmo llevada de lo local a lo nacional, cuyo propósito era el autogobierno y el mayor acercamiento posible de la voz popular a las decisiones gubernamentales (Marti, 2006). Los principales temas de reflexión entre los años sesenta y setenta, consistirá en los mecanismos de participación como son: revocatoria del mandato, la consulta popular, el plebiscito, el 
referendo, la iniciativa popular, el cabildo abierto, entre otros; con participación cuantitativa se buscaba corregir en altos índices la ilegitimidad de la democracia representativa y, por esto, también va a ser conocida como democracia refrendaria (Mejía y Blanco, 2005).

\section{LA LLEGADA DE LA DEMOCRACIA DELIBERATIVA}

Para la década de 1980 aparece el concepto de democracia deliberativa, la cual venía hacer una nueva forma de democracia, aclarando por supuesto que la democracia deliberativa no se concibe como la practicada en la antigua Grecia, mientras que "la democracia representativa viene funcionado por más de 200 años y la democracia participativa en su versión decisional y deliberativa en los últimos 50 años, mientras que la republica deliberativa se considera una utopía casi imposible en una sociedad moderna" (Martí, 2006, p. 14).

La democracia deliberativa fundamentada en el intercambio de información y mecanismos participativos abren la posibilidad de la deliberación, la cual facilita a los ciudadanos generar una mejor decisión en contra de las decisiones tomadas en los mecanismos de participación directa, esto implica una forma de gobierno en la cual confluyen la representación con mecanismos de participación deliberativa, bajo principios de la argumentación y comunicación. Entre los más importantes exponentes de este modelo democrático que se fundamenta en "la razón" y "la comunicación" se encuentran Jon Elster, Jürgen Habermas, Joshua Cohen, Carl Sustein, entre otros.

A través del debate y la discusión, el pueblo, la economía y la sociedad civil generan consenso y decisiones mejor sustentadas y garantistas (Dahl, 2003). En otras palabras, aunque la participación es importante, no interesan tanto el número de votos y el censo electoral como ocurre con la democracia participativa decisionista, sino que la gran preocupación ronda por el intercambio de información, el debate generado a partir de éste y como consecuencia la toma de mejor decisión.

\section{LA DEMOCRACIA PARTICIPATIVA}

Entrando a los años 90, la democracia participativa se debate entre el modelo decisional y el deliberativo, no quedando claro los procedimientos que atañen a cada uno de estos, es así como en los diversos países que asumen las reformas de sus Cartas Políticas se convienen los mecanismos de participación sin que se resuelva el dilema entre los dos modelos de democracia participativa a los que nos hemos venido refiriendo.

Actualmente, el recurso de apelar a los mecanismos de participación decisionista validos de los medios de comunicación y a las crisis de legitimidad de las democracias representativas ha venido incrementándose, dando lugar a que los sis- 
temas presidencialistas frente al desprestigio de los legisladores, máximo ente de representación popular, acudan a la sociedad para legitimar sus políticas de Estado por medio de asambleas constituyentes, referendos y plebiscitos.

La falta de deliberación ha permitido la manipulación de la implementación de mecanismos de participación directa, en el sentido de que las mayorías logran sus cometidos a costa de las minorías porque finalmente al no existir la suficiente información y deliberación de los temas a decidir, terminan siendo sometidas las minorías con medidas autocráticas que cuentan con respaldo de las bases populares y en algunos otros, por una sólida estructura burocrática y el respaldo de los medios de comunicación.

EVOLUCIÓN HACIA LA DEMOCRACIA PARTICIPATIVA EN COLOMBIA

En materia de transformaciones institucionales, Colombia requería, entre otros países de América Latina, modernizarse como Estado, lo que implicaba entre otros cambios, acercar la sociedad al Estado y éste actuar en consenso con las demandas de los individuos y de la sociedad en general, a fin de garantizar los derechos fundamentales como los sociales, económicos y culturales. Este nuevo pacto fundacional en palabras de Uribe (2002), se fue configurando en torno a la democracia participativa; ésta fue vista como la "clave y el hilo conductor de la Carta Constitucional".

En un somero análisis del paso de una democracia representativa a una democracia participativa se debe tener en cuenta el quiebre ideológico de 1970 que plantea el constituyente Orlando Fals Borda (1991) cuando afirma que había necesidad de repensar los problemas organizativos de los esquemas vanguardistas de los socialistas sectarios frente a la democracia burguesa, acompañado del fracaso del desarrollo propuesto por los países avanzados, pero que en nuestros países no alcanzaban los logros esperados.

Es así como la polarización entre los grupos armados en Colombia (FARC, EPL y ELN) y el bipartidismo tradicional (Partidos Liberal y Conservador), ponen en cuestión los problemas de la sociedad, simultáneamente movimientos políticos alternativos como el Gaitanismo, la ANAPO que da lugar a la creación del Movimiento Guerrillero 19 de Abril (M-19), los campesinos representados por la Asociación Nacional de Usuarios Campesinos (ANUC), que organizó tomas masivas de tierras, de igual manera, los indígenas se organizaron de manera autónoma con el Consejo Regional Indígena del Cauca (CRIC) y los movimientos estudiantiles constituyen el cuadro de la conflictividad del Estado colombiano en oposición contra el bipartidismo tradicional, sin que se lograran inicialmente avances hacía una propuesta de construcción de mecanismos de participación democrática por las dificultades presentadas en el seno de la misma izquierda. A finales de la década de los setenta, como lo señala Borda (1991), con el aporte del pensamiento de intelectuales como Estanislao Zuleta y algunas fundaciones, impulsaron el pensamiento de marxólogos críticos como Lucio Colletti y publicaron en 1977 las conclusiones de un simposio mundial sobre investigación-acción, donde se recuperó la figura alterna de Antonio Gramsci y se sentaron bases para el trabajo de movimientos sociales populares 
y regionales que permitió a la izquierda seguir trabajando por la reconstrucción nacional y la apertura democrática, iniciativas que fueron luego acogidas por la Asamblea Nacional Constituyente como son la elección popular de los alcaldes y gobernadores, la soberanía del pueblo y la participación de éste a través de plebiscitos, referendos, el respeto por la diversidad étnica, la defensa ecológica y la integración de América Latina (Borda, 1991).

La consagración definitiva del principio fundamental del modelo de democracia participativa en la Constitución colombiana de 1991, junto con los diversos mecanismos de participación, no permite vislumbrar si se trata de un modelo de democracia participativa decisional o deliberativa, lo que genera una gran incertidumbre para los diversos sectores sociales excluidos tradicionalmente en la historia colombiana y que esperaban participar no sólo en las decisiones y deliberaciones acerca de sus derechos fundamentales, sociales, económicos y culturales, sino que seguramente podrán ser objeto de las decisiones autocráticas, donde impera un fuerte presidencialismo y que seguramente buscan beneficiar a las clases con mayor poder económico y político.

\section{DEMOCRACIA PARTICIPATIVA Y GOBERNANZA DEL AGUA}

\section{La PARTICIPACIÓN}

Si bien, la participación tiene lugar en las transformaciones de las tipologías de la democracia, se reivindica el tema del contexto planteado por Baños (2006), Sartori (1994), Dahl (1992), Goodwin (1997), para quienes en general, cada periodo histórico genera nuevas corrientes de pensamiento según las experiencias y nuevas preguntas a resolver y asigna a cada uno unas características específicas. Pero también, el contexto aplica para el concepto de participación y de ciudadanía, pues como lo explica Sorj (2005), "el desafío que el concepto de este último plantea consiste en distinguir entre el significado asignado por el sentido común, con una fuerte carga normativa, y una noción más rigurosa, provista de un valor empírico-analítico" (pp. 20-21). Es así como, según Sandoval (2013), la manera actual como se conoce la participación comunitaria no siempre ha sido igual [...], hasta los años setenta del siglo xx la participación era una herramienta "para lograr la sumisión voluntaria de la gente a los esquemas de áreas protegidas" (p. 88), y por ende, la población local era vista como un actor pasivo. En los años ochenta se definía por el interés sobre la protección del recurso natural; a partir de los años noventa, se plantea la participación como el hecho de incluir a los ciudadanos en la gestión del área protegida.

Para él, el primer paso a dar para deslindar el concepto de ciudadanía consiste en insertarlo en la dinámica de cada sociedad históricamente determinada, sin embargo, advierte sobre el riesgo de caer en el relativismo y reconocer la existencia de una infinidad de "ciudadanías", para lo cual reclama la importancia de los análisis teóricos y comparativos, que pueden contribuir a identificar "los componentes básicos comunes" que constituyen cada uno de esos conceptos. No obstante, afirma que "la ciudadanía es, por tanto, un concepto, resbaladizo, una especie de "bisagra", que se sitúa en el punto de encuentro entre el individuo y la comunidad, siendo el 
mecanismo que permite al individuo reivindicar su condición singular o su libertad personal y a la vez afirmar su pertenencia al grupo" (Sorj, 2005, p. 24).

Sorj recuerda, que en el contexto de las teorías de la democracia la ciudadanía, se reconoce como una realidad histórica que se transformó a medida que pasaba el tiempo, pero que según Marshall, se originó a partir del ideal de igualdad de la esfera jurídica hacia la esfera política y social, pero que además son los principios de "comunidad e individuo" los que sostienen las dos ideas fundadoras de la ciudadanía moderna: la soberanía de pueblo y la igualdad de los ciudadanos ante la ley rompiendo con el pensamiento tradicional que pregonaba que el poder temporal provenía de la voluntad divina (...).

En este sentido, es preciso reconocer el rol transformador de la ciudadanía, una vez adhiera a un proyecto colectivo en el que pueda co-crear o ayudar a construir unas nuevas relaciones entre la sociedad y el Estado, fortaleciendo así el sentido de la democracia. Es así como Peters (2007) acuña el término "gobernanza democrática", enfatizando en que más allá de la preocupación por "la capacidad de tomar decisiones", es necesario preocuparse por "cómo se toman esas decisiones" (p. 4), y cuáles actores dirigirán el rol funcional para la sociedad. En razón a ello, defiende la constitución de redes como mecanismos de gobernanza que favorecen las estructuras más deliberativas y la movilización del proceso de elaboración de políticas públicas, pese a reconocer que no se logrará un proceso del todo democrático y deliberativo.

Con respecto a la participación en el marco de la democracia, cuando los autores se refieren a los discursos y a las palabras, es preciso atender a lo expuesto por Lindblom, con respecto al proceso de elaboración de las políticas públicas, quien afirma que 'dada toda la organización del Legislativo, el liderazgo del Ejecutivo, y los grupos legislativos, y consideradas las opciones que tienen los decisores de políticas públicas, es evidente que no hay una relación clara entre lo que desean y las políticas que obtienen los electores' (Lindblom, 1991). El diseño y formulación de estas políticas obedecerán a la estructura de las normas, de las relaciones de autoridad, de los procedimientos y de las organizaciones que medien entre ellos y el efecto que ejerzan en las políticas. Mientras tanto, la pregunta es idónde queda el control popular dentro de un sistema democrático?, pues, si bien es cierto que los aspectos anteriormente expuestos no 'mutilan la democracia, si la debilitan (...). Las normas de la democracia entregan importantes poderes y libertades a los ciudadanos, pero solamente les confiere un control débil sobre las políticas (Lindblom, 1991, p. 82). A partir de estos planteamientos, se tendrán en cuenta los aportes que desde la teoría política se hacen en cuanto a la democracia y a la democracia deliberativa.

LOS ESPACIOS DELIBERATIVOS

De acuerdo con lo planteado por Cohen (2013, citando a Fung \& Wright, 2001), la evolución de las teorías de la democracia representa en cierta medida, la evolución del Estado y por tanto se afirma que en la medida en que algunas funciones del Estado han evolucionado a partir de las formas de democracia liberal del siglo XIX, en tanto los mecanismos tradicionales de representación política, no logran satisfacer 
muchos aspectos de la vida ciudadana del siglo Xxi. Para esta autora, "la deliberación permite repensar nuevos acuerdos de gobernanza que incrementan la participación y la deliberación misma, tanto en la esfera del mercado como en las del gobierno y la sociedad civil", y en este sentido, la democracia deliberativa conlleva a la acomodación de las fuerzas políticas y sociales "que permite a los ciudadanos participar e influir sobre las decisiones que afectan sus vidas [...], la exploración de la deliberación es una estrategia progresiva de reforma institucional que permite avanzar en el entendimiento empírico y conceptual de la práctica democrática, pues pone en juego, los valores de participación, deliberación y empoderamiento bajo límites de prudencia y viabilidad" (Cohen, 2013).

$\mathrm{Al}$ respecto, a partir de los en los postulados de la democracia deliberativa, expuesta por autores como Jurgen Habermas, Joshua Cohen, James Bohman y Jon Elster, que presentan la democracia deliberativa como una visión explícitamente normativa de la democracia, cuyo núcleo central es la legitimidad de los procesos democráticos, Hevia (2011) acude a la definición del concepto de Elster, para quien la democracia deliberativa "[...] incluye la toma colectiva de decisiones con la participación de todos los que se verán afectados por la decisión o la de sus representantes: esta es la parte democrática. Además [...] incluye la toma de decisiones por medio de argumentos ofrecidos por un par de participantes a los demás, comprometido con los valores de la racionalidad e imparcialidad: ésta es la parte deliberativa". Con base en esta definición el autor asume que la democracia deliberativa pretende articular los ideales de participación, razonamiento y legitimidad política. Es por medio de la argumentación y el razonamiento público y de la participación de los diversos intereses en juego, que la norma adquiere su legitimidad, en la medida que los actores están en igualdad de condiciones.

La deliberación constituye entonces, un mecanismo fundamental para la toma de decisiones y reitera los tres principios fundamentales, que modifican la práctica política, en tanto devuelven autoridad a la localidad, incrementando la importancia de la vida cívica y sus múltiples actores para generar una democracia más vigorosa y eficaz; crean vínculos formales de responsabilidad ciudadana, distribuyen recursos e información para articular a los diversos actores entre sí y con las autoridades, contribuyendo de esta manera a la creatividad en la edificación de las nuevas instituciones democráticas con mayores márgenes de participación y eficacia frente a la formas tradicionales de representación política y administración burocrática. Estos principios son:

a. Un enfoque centrado en los problemas concretos y tangibles;

b. co-participación del ciudadano común afectado por determinadas problemáticas y las autoridades involucradas, y

c. desarrollo del giro deliberativo para la búsqueda de soluciones comunes.

Teniendo en cuenta que la presente investigación se intenta analizar la participación ciudadana en el proceso de gobernanza a escala local, es necesario también recurrir a lo que se entendería, específicamente por participación, la cual según Landázuri (en Gatica et al., 2008) para algunos autores es "sinónimo de consenso y asistencia; para otros implica dar e intercambiar información, y aceptación de decisiones tomadas por otros o en otros espacios (o) capacidad de formular, ejecutar, planificar, seguir y evaluar diferentes estrategias" (p. 240).

En cuanto a autores latinoamericanos, Oszlak (2009), por ejemplo, cuestiona las categorías dentro de las cuales se enmarca el término participación', y afirma que 
"la propia naturaleza polisémica del término que adjetiva esa participación (ciudadana, popular, social, cívica, comunitaria) es una primera indicación, sin entrar a considerar las dificultades que todavía enfrenta la definición de lo que debemos entender por 'sociedad civil".

\section{Por El RECONOCIMIENTO DEL DERECHO HUMANO AL AGUA}

Si bien, la aprobación de la Constitución Política de 1991 y con ella, el reconocimiento de la existencia de la democracia participativa en Colombia como principio afianzó ideas propuestas por los grupos mencionados supra, cuyos fines políticos estarían claramente determinados, también propició el surgimiento de una serie de organizaciones y mecanismos que favorecieron el reconocimiento de otros derechos como la defensa ecológica y que en su momento buscaron ser incorporados dentro de los derechos fundamentales tales como el derecho humano al agua.

El derecho humano al agua (y al saneamiento) fue reconocido formalmente el 28 de julio de 2010, a través de la Resolución 64/292, la Asamblea General de las Naciones Unidas, aunque el año 2002 "ya lo había reconocido en su observación No. 15 en la interpretación de los derechos económicos y culturales" (Motta, 2013, p. 165). Sin embargo, para el caso colombiano, el antecedente más cercano se remonta al año 2007, en donde a raíz de la dificultad para acceder al líquido por factores como el aumento de la población, la contaminación, la deforestación y el calentamiento global (Motta, 2010), surge la iniciativa de referendo por el derecho humano al agua y al mínimo vital gratuito. De ello se encargó el "Comité Promotor del Referendo por el Derecho Humano al agua" con el fin de presentarlo como proyecto de ley ante el Congreso para que fuera incluido dentro de la Constitución Política. Se fundamentaba en cuatro aspectos fundamentales: a) el acceso al agua como un derecho fundamental, b) la garantía de un mínimo vital gratuito para los hogares colombianos, c) la no privatización de los servicios de acueducto y d) la garantía la conservación del medio ambiente en el suministro del agua global (Motta, 2010, p. 258).

Sobre la Resolución de la onU, se afirma que hubo un trabajo previo de la sociedad civil global para hacer campañas de sensibilización e incluso hicieron borradores de la normativa (Marín, 2010), por ende, la declaración del Derecho Humano al Agua es un logro de las luchas sociales por la identidad y el territorio local (Boakye y Akpor, 2012). No obstante, y en contraste con lo establecido por la ONU, y de acuerdo con Marín (2010), en Bolivia distintos actores de la sociedad global denunciaron los alcances de la ley 2029 de 1999 que vendería el agua dejando de lado los sistemas comunales, los cuales sin obligación estaban vinculados a los sistemas de agua potable municipal. Para ello, organizaron la Coordinadora para la Defensa del Agua y de la Vida que realizó tanto protestas como investigaciones sobre toda la problemática y los actores (el consorcio, funcionarios) que la promovían; los medios de comunicación reportaron la situación como "la guerra del agua". De manera que después de tanta presión y entrar en negociación con el Estado, se logró que la ley fuera derogada, se liquidara la empresa operadora, liberaran a los manifestantes detenidos y el agua se normalizara de acuerdo con los usos tradicionales. 
Esta concepción sobre el agua como derecho humano, es la que prevalece en enfoques de justicia del agua, entendida como "bien común" propuesto por Barlow (2001), y quien en su calidad de asesora del Presidente de la Asamblea de la ONU, inspiró la resolución 64/292 de 28 de julio mencionada supra, complementada con la aprobada el 10 de septiembre de 2010, que agrega que éste "es igual a todos los demás derechos humanos, lo que implica que es justiciable y de aplicación obligatoria" (Colmenares, 2013). En este enfoque predomina el elemento participativo comunitario, como aspecto fundamental para hacer efectivas la justicia y democracia del agua.

En contraposición a éste se encuentra el concepto de injusticia ambiental, entendida como la inequidad en la distribución de los daños ambientales sobre poblaciones de diferentes condiciones socioeconómicas (Alves, 2007, citado por Fracalanza, A.; Martins Jacob, A. y Furtado Eça, R., 2013) y por la desigualdad en el acceso a los recursos ambientales, reforzando la relación entre riesgos ambientales y desigualdades socioeconómicas (Veiga, 2009). En este sentido, Fracalanza et al., acuden a Porto (2004), para quien la injusticia ambiental es el mecanismo por el cual sociedades desiguales, desde el punto de vista económico y social, otorgan lugares con mayor carga de daños al medio ambiente, a poblaciones de bajos recursos, grupos sociales discriminados, tradicionales pueblos étnicos, barrios marginados y poblaciones vulnerables de los trabajadores (Porto, 2004, p. 122, citado por Fracalanza et al., 2013).

Algunos enfoques conceptuales sobre la gobernanza del agua

Varios de los autores revisados optan por acoger propiamente el término gobernanza del agua, y en este sentido, identifican diversas posturas teóricas o conceptuales para definirla, advirtiendo que no hay una definición universal, por su carácter multidimensional (Valdovinos, 2011), multidisciplinario (Araral y Wang, 2015), que continúa en negociación (Ashtana, 2013) y sobre el cual no se ha llegado a un consenso sobre su definición o aproximación conceptual (Araral y Wang, 2015).

De acuerdo con Lemos y Agrawal (2006), el concepto de gobernanza puede ser observado bajo cuatro dimensiones: globalización, gobernanza ambiental descentralizada, instrumentos enfocados en el mercado y los individuos, y gobernanza a través de escalas. La primera dimensión es la globalización de problemas medioambientales que han permitido la creación de organizaciones, instituciones y regímenes que funcionan a nivel global y amplían el rol de los movimientos sociales. Además de innovar en las estrategias y las soluciones y movilizar la opinión pública, promueve la cooperación entre actores no estatales como "intereses corporativos, movimientos sociales y organizaciones no gubernamentales" (Lemos y Agrawal, 2006, p. 301) que han estado usualmente por fuera del proceso de la política.

La segunda dimensión es la gobernanza ambiental descentralizada, "cuya efectividad depende de altos niveles de participación y mayor involucramiento de ciudadanos en el proceso de gobernanza" (Lemos y Agrawal, 2006, p. 303), de manera que se confía en las comunidades, como los grupos de usuarios de agua y las formaciones sociales a pequeña escala para organizarse y manejar los recursos hídricos o ambientales (gestión de los recursos naturales basada en la comunidad) y se 
transforma la gobernanza hacia una cogestión y descentralización de la política ambiental. Lo anterior resignifica y descentraliza la gobernanza al plantearla por fuera del Estado o instituciones de libre mercado, justificándose en que puede aumentar la eficiencia. El proceso de decisión está más cerca a quienes se ven afectados, hay "mayor participación y responsabilidad. Finalmente ayuda a quienes toman las decisiones (decision makers) a tomar ventaja de un conocimiento más preciso en las especificidades de tiempo y de espacio de los recursos naturales" (p. 303).

Muchas investigaciones se han centrado en la relación de abajo a arriba (respecto a quienes toman la decisión en el nivel inferior y superior) y en cómo se relacionan quienes toman las decisiones con sus electores, dejando de lado el cambio en las relaciones subjetivas entre los sujetos y el medio ambiente según la transformación de las relaciones de poder y gobernanza. En la tercera dimensión los autores se enfocan principalmente en los actores corporativos, en una relación de costo-beneficio con estrategias ambientales particulares para así movilizar incentivos individuales en favor de resultados ambientales positivos.

La última dimensión de Lemos y Agrawal (2006) son los mecanismos de gobernanza a diversas escalas que se expresan a través de actores no estatales como ONGS, organizaciones medioambientales transnacionales, organizaciones intergubernamentales y multilaterales, actores orientados por el mercado (multinacionales y transnacionales) y comunidades epistémicas, quienes se construyen como nuevos actores que introducen "herramientas y mecanismos innovadoras y configuran positivamente las relaciones de poder en la arena política, incluso si su potencial transformador es debatido" (p. 309). Complementando esta forma de gobernanza, Brondizio, Ostrom, Young, (2009) plantean la gobernanza multinivel, que consiste básicamente en sistemas de gobernanza que son compatibles con otros sistemas y deben incluir mecanismos para aliviar las tensiones entre los niveles individuales según sus circunstancias y necesidades (Brondizio et al., 2009, p. 269).

Lemos y Agrawal (2006) consideran que la gobernanza está siendo reconfigurada por la globalización y la descentralización, así surgen formas institucionales alternativas, creadas por la convergencia o la acción de Estado, mercado y/o sociedad civil o comunidad; teniendo en cuenta que la inclusión de la comunidad como actor es más reciente. Entre los ejemplos que atribuyen a cada forma de gobernanza, el agua se encuentra entre el comanagement (relación entre agentes del Estado y comunidad), las otras dos relaciones consisten en "asociación público-privada (entre agencias estatales y actores del mercado), y asociación socio-privada (entre actores del mercado y comunidades)" (Lemos y Agrawal, 2006, p. 311). Los autores resaltan que algunas investigaciones presentan formas híbridas donde se apelan a varios de los mecanismos anteriores para enfrentar los problemas ambientales, dada su complejidad.

Sin embargo, alterando un poco el orden propuesto por Lemos y Agrawal (2006) y Brondizio et al., 2009), la siguiente clasificación pretende reorganizar en cuatro grandes grupos los hallazgos la información revisada, no sin antes advertir que algunos autores aportan con sus discusiones a los diferentes grupos. Primero, aquella que destaca aspectos relacionados con las instituciones y la burocracia ligados a las distintas fases del ciclo de las políticas públicas. Segundo, la que privilegia la intervención de los ciudadanos en calidad de usuarios, afectados o beneficiarios, en enfoque de corte más participativo, sin desconocer el papel del gobierno y sus instituciones. Tercero, aquella que centra su discusión en los aspectos territoriales para el manejo del agua. Cuarto, la centrada en la gobernanza global o multinivel. 


\section{GOBERNANZA DE AGUA Y MANEJO AMBIENTAL}

En el primer grupo, uno de los intentos consiste asociar la gobernanza del agua, como sinónimo de manejo ambiental del agua, que incluye dentro de sus tareas formular, planear e implementar políticas públicas fundamentadas en el conocimiento científico, los valores de la comunidad, los distintos niveles de riesgo que presentan los cuerpos hídricos y establecer alianzas entre los actores involucrados; a fin de monitorear, reportar, evaluar y mejorar dichas políticas públicas y el régimen de gobernanza elegido (Hornel y O’Donnell, 2014). Esta línea de gobernanza del agua favorece el manejo sistemático, burocrático o institucional.

Ünver (2008) y Ashtana (2013) usarán una definición similar al proponerla como un "rango de sistemas políticos, sociales, económicos y administrativos usados para desarrollar y manejar los recursos hídricos y acceso a los servicios del agua en los distintos niveles de la sociedad". Mientras que Paerregaard (2013) se refiere a la gobernanza del agua como "el manejo burocrático de la infraestructura hídrica y el control social de la distribución del agua dentro de un sistema de irrigación" (p. 207). De manera complementaria, Ruiters y Matji (2015) comprenden la gobernanza como actores (principalmente instituciones) que manejan el agua o como serie de estrategias o instituciones para recaudar dinero y así mejorar y desarrollar la infraestructura hídrica.

\section{LA CO-GOBERNANZA DEL AGUA}

Quizá sea pertinente mencionar el enfoque para el cual la concepción del manejo del recurso se aborda desde la co-gobernanza del agua. Su aplicación estratégica tuvo lugar a partir de una decisión política del gobierno de una provincia china a través de un programa denominado Co-gobernanza de las Cinco Aguas para el tratamiento de aguas residuales, prevención de inundaciones, drenaje, abastecimiento y conservación del agua, dentro del cual se señala un sistema de compensación por el uso (adecuado) del recurso hídrico y su impacto positivo en los ecosistemas alrededor, para así constituir una civilización ecológica. Si bien, en este enfoque las directrices vienen dadas desde el gobierno, en dicha civilización se establecen los límites necesarios y se transforma el comportamiento humano para ejercer la protección ecológica.

Este tipo de modelo de gobernanza refleja "la comprensión exacta de los requerimientos del desarrollo social" para mantener el agua asequible y limpia, tomando en consideración un equilibrio entre los recursos hídricos, la capacidad de abastecimiento, el consumo del agua, la emisión de contaminantes desde todos los sectores (agricultura, industria, servicios, entre otros), la capacidad de tratamiento de las aguas residuales y de carga ambiental (Xiang, 2015, p. 805). 
Al respecto, el enfoque del buen gobierno o la governance, y como lo reitera Ruano (2011) refiriendo el caso de Guatemala, los gobiernos locales y centrales no deberían eliminarse de la gobernanza del agua por la existencia de proyectos basados en la comunidad que resultan exitosos en ciertos contextos, ya que ellos son quienes aseguran y protegen el agua como derecho. Este autor en particular convoca a que el Estado esté dispuesto a "tener políticas más comprensivas y asignar responsabilidades más claras para todas las partes involucradas" y así evitar la situación histórica que pone a poblaciones rurales y pobres a buscar por ellos mismos cómo acceder al agua (Ruano, 2011, p. 8).

Desde un enfoque de control social, otros autores hablarán de gobernanza como accountability o rendición de cuentas (Hill, Furlong, Bakker, y Cohen, 2008). En relación con este enfoque está la participación social, y en él, la gobernanza del agua es entendida como coordinación, gobierno y manejo del agua a través de múltiples niveles (Grecksch, 2015; Valdovinos, 2011), en donde se favorece la información que circula entre dichos niveles, y se evidencia un incremento de la participación por grupos de la sociedad civil y usuarios del agua, que no sólo pertenezca al sector político (Grecksch, 2015).

Es aquí en donde la gobernanza como alternativa más participativa, puede entenderse como gobernabilidad, en la que "se toman decisiones dentro o entre organizaciones, incluyendo: actores involucrados, asignación de responsabilidades, priorización de los objetivos y la rendición de cuentas", a través de acuerdos, procedimientos, convenciones y políticas, lo que en un nivel institucional correspondería al análisis de las reglas de juego, pero buscando siempre el balance de poderes y de acciones de acuerdo con cada nivel de autoridad (Daniel, Pinel, y Brooks, 2013). La asignación de responsabilidades debería entonces distribuirse dentro de múltiples actores claves (Zhou, Du y Bai, 2015).

No obstante, las reglas de juego no estarían regidas sólo por un marco legislativo del agua (Daniel, Pinel y Brooks, 2013); acuerdos contractuales o programas de participación entre Estados, municipalidades, y operadores privados (Schneier-Madanes, 2014); sino también por las reglas informales, como son las prácticas culturales, acuerdos, consensos, usos, costumbres (Daniel, Pinel, y Brooks, 2013), guerras del agua, protestas y competencias políticas (Schneier-Madanes, 2014). Esto implica ampliar los mecanismos de la gobernanza y trascender la respuesta técnico/ legal (Domínguez, 2010); lo que se denominaría redes formales e informales entre agencias y el diseño de marcos legales y de tratados (Daniel, Pinel y Brooks, 2013).

En consecuencia, la gobernanza puede ser entendida como un conjunto de procesos formales e informales o proceso de interacción y de negociación de intereses, similares u opuestos; que involucra a actores heterogéneos, numerosos y claves, esencialmente públicos o estatales, sociales y privados, cuya interacción se da a partir de redes o estructuras de relación para determinar la forma y las modalidades de toma de decisiones y ejercicio del poder (Brenner y de La Vega, 2014; Ruiz y Gentes, 2008; Valdovinos, 2011; Daniel, Pinel, y Brooks, 2013).

Los actores claves pueden ser definidos como personas u organizaciones afectadas o que tienen la capacidad de influenciar sobre el asunto hídrico (Benhamt, Hussey, y Beavis, 2014), cuyos participantes son llamados según su grado de representación de un grupo clave; principio aplicado usualmente en las políticas hídricas, 
forestales y de pesca dentro de un modelo de gobernanza regional con enfoque de democracia representativa, el cual funciona siempre y cuando los mecanismos estén bien diseñados y el gobierno pueda usar el conocimiento local para "promover la democracia, aumentar el apoyo, tomar en cuenta valores, resolver conflictos y crear condiciones para lograr como resultado una política justa" (Howard, 2006, p. 254).

Es importante resaltar "que los Estados no son los únicos actores que poseen 'poder agencial", por lo cual resulta importante incluir otros "individuos, comunidades rurales, y líderes tribales" o los denominados grupos o comunidades de interés, a fin de resaltar los procesos interactivos y "considerar la dimensión normativa de interacciones como creencias y valores” (Meissner, 2014, p. 2). Se entiende el poder agencial como la capacidad de "dar a los actores agencia para influenciar su medio y a cada uno de los actores", poder que también puede ser reflexivo, lo cual indica que su capacidad de gobierno y poder aumentarán (Hobson citado en Meissner, 2014, p. 2); y que puede ser ejercido tanto a nivel relacional como estructural. Dentro del poder agencial, intervendrán la ideología, el poder económico y político. La interacción entre tanto se caracterizará por el conflicto, la confrontación, la cooperación o la adaptación, el control o la desviación, la cual deberá ser siempre contextualizada (Meissner, 2014).

La propuesta anterior, según el autor, ayudará a construir un pensamiento más sistemático sobre las políticas y gobernanza del agua. Sin embargo, la gobernanza no sólo es una forma de direccionar la política y el manejo del agua al analizar la interacción y los sistemas sino que también requiere del análisis de las prácticas y discursos (Schneier-Madanes, 2014).

UNA MIRADA DESDE EL NIVEL TERRITORIAL

Si bien, los autores antes mencionados han hecho referencia a los niveles local, nacional, internacional y transnacional para abordar el análisis de la gobernanza del agua, algunos como Ruiz y Gentes (2008) se han centrado exclusivamente en el nivel territorial, particularmente en la cuenca hidrográfica. Ésta constituye el límite geográfico y social para analizar la gobernanza del agua y por ende, implementar mecanismos participativos e institucionales. Se fundamentan en argumentos hidrológicos y socioeconómicos, entendiendo este tipo de gobernanza desde dos direcciones: desde las políticas públicas del Estado, respetando y haciendo respetar la autonomía de derecho consagrada a favor de los actores locales en el interior de las 'cuencas sociales'; y desde las propias comunidades y municipios, acompañando y apoyando la autonomía y poder de decisión otorgado, por ejemplo, en el manejo de redes de riego, el suministro del agua y la conservación de los recursos naturales (Ruiz y Gentes, 2008, p. 45).

La gobernanza a nivel de cuenca hídrica buscaría entonces un balance entre un manejo centralizado y local, al tener una multiplicidad de actores en cada nivel jurisdiccional (Horne y O'Donnell, 2014); ello significaría aceptar que existe una territorialización del agua y se ha convertido en una categoría de análisis de la acción pública y que el objetivo del nivel local es asegurarse de que los consumidores en un territorio determinado pueden dialogar el uno con el otro a través de contratos enfocados en ríos, golfos, lagos o capas freáticas (Schneier-Madanes, 2014). 
Otra propuesta ha sido tomar como unidad de estudio, la subcuenca o la región, que incluso se califica como efectivo, como también se podría combinar la región administrativa con la cuenca y así mejorar el concepto de conservación del agua (Xiang, 2015). No obstante, recientemente ha habido un cambio conceptual de gobierno a gobernanza, lo cual indica que ya no existe un único actor que decide y tiene control sobre la sociedad civil, sino que la gobernanza ya implica "una condición multinivel, policéntrica, donde varios actores en configuraciones institucionales diferentes contribuyen al desarrollo e implementación de la política" y por eso tiene en cuenta actores gubernamentales y no gubernamentales (Pahl-Wostl, Gupta, y Petry, 2008b, p. 423). En ese sentido, gran parte de la literatura revisada aborda la noción de gobernanza global del agua como respuesta al ciclo hidrológico que incluye comunidades, Estados y cuencas y en especial, al mundo como un todo (Darling, 2012; Schneier-Madanes, 2014).

La llegada de un nuevo contexto económico, político y cultural a nivel global, trae consigo nuevos y grandes conflictos, nuevas formas de manejar los sistemas, sus funciones, las políticas públicas y las condiciones de vida e incluso, los cambios en la naturaleza (Schneier-Madanes, 2014) que requiere comprender la gobernanza del agua a nivel global, denominada también gobernanza muktinivel, contrario a lo que proponen otros autores como Ruiz y Gentes (2008) y Horne y O'Donnell (2014).

Este enfoque plantea que el sistema hidrológico es global, cuyos procesos de intercambio ocurren en periodos relevantes (por ejemplo, los impactos del cambio climático); las fuerzas motoras de los problemas del agua yacen por fuera de los regímenes usuales de gobernanza como consecuencia de los fenómenos socioeconómicos y el cambio ambiental a nivel global; y las consecuencias de la reducción de la calidad o cantidad del agua son de carácter global (migración de pájaros, cambio en los sistemas productivos del agro) (Pahl-Wostl, Gupta, y Petry, 2008b, p. 422).

En este sentido, Darling (2012) plantea que existe al menos un "naciente sistema de gobernanza", el cual según Pahl-Wostl, Gupta, y Petry (2008b), "tiene un carácter multinivel y los mecanismos globales deben incorporarse en modos que sean complementarios a instrumentos aplicados en otros niveles". Los autores explicarán que la gobernanza global del agua es "el desarrollo e implementación de normas, principios, reglas, incentivos, herramientas informativas e infraestructura para promover un cambio en el comportamiento de los actores en el nivel global, en el área de la gobernanza del agua" (p. 422). Ésta será promovida o ejercida por instituciones políticas (Ashtana, 2013) o por un rango amplio de actores claves (Schneier-Madanes, 2014), que "buscan coordinar las relaciones independientes e interdependientes", las cuales pueden ser de carácter formal, informal, estricta, flexible, permanente, transitoria, pública o privada" (Ashtana, 2013, p. 63). Así mismo, pueden presentar desconexiones y conexiones en los distintos niveles (Scheneier-Madanes, 2014).

El reconocimiento de la dimensión global del recurso hídrico sirve como metáfora del campo del agua en la cual "estrategias concretas de diferentes actores definidos por su posición, propiedades e intereses interactúan" (Schneier-Madanes, 2014 , p. 6). Ello requiere la inclusión de una perspectiva global en todos los niveles de la gobernanza y la adopción de sistemas multinivel de gobernanza, entendidos como el diseño simultáneo de políticas hídricas a escala global, fluvial, nacional, provincial y local (Pahl-Wostl, Gupta, y Petry, 2008b, pp. 422-423). Para el logro de la red de la política global del agua y la construcción de un consenso alrededor de ésta, se requiere "formación, seminarios, talleres, educación, difusión mediática del conocimiento" (Ashtana, 2013). 
De modo que al hablar de una gobernanza multinivel o de gobernanza global del agua, los sistemas de gobernanza se complejizan, lo cual requiere de instituciones y prácticas de gobernanza ejercidas a un nivel internacional, nacional (Mumme, Ibáñez, y Till, 2012), local y regional, en relación también con las dinámicas intercomunales y la toma de decisiones en los territorios (Scheneier-Madanes, 2014). La gobernanza multinivel se puede comprender entonces como sistemas poliárquicos, dentro de los cuales "distintas formaciones sociales, gubernamentales, no gubernamentales, formales e informales a varias escalas y diferentes niveles de complejidad, podrían mantenerse en sistemas de gobernanza prácticos y relativamente estables en ciertas circunstancias" (Ostrom citada en Mumme, Ibáñez, y Till, 2012, p. 7).

El enfoque multinivel se puede clasificar en dos tipos, tipo I que consiste en "instituciones de gobierno tradicionales en multiniveles y entidades de gobernanza más discretas funcionalmente y particulares encontrándose en diferentes escalas y jurisdicciones sociopolíticas". El tipo II "contribuye a la flexibilidad institucional, solución transnacional de problemas y construcción de relaciones (...) que debería aumentar la cooperación social y reducir el conflicto a través de una colaboración internacional más efectiva" (Hooghe y Marks citados en Mumme, Ibáñez y Till, 2012, p. 7).

Por otro lado, ha habido un intento de clasificar y distinguir entre jerarquía, redes y mercados como modos principales de gobernanza, pero estos pueden interrelacionarse, encontrando que "los mercados y las redes funcionan mejor dentro de un marco regulatorio oficial donde los contratos y el reino de la ley son respetados" (Pahl-Wostl, Gupta, y Petry, 2008b, p. 423). Sin embargo, el desarrollo de la gobernanza global del agua depende de las tensiones entre globalización y regionalización; centralización o descentralización; procesos y resultados formales o informales; la influencia del estado vs actores y procesos no estatales (Pahl-Wostl, Gupta, y Petry, 2008b, p. 424).

En ese sentido, algunos modelos de gobernanza para recaudar dinero parten directamente del nivel nacional o local y del orden principalmente público o sus combinaciones entre entidades privadas y públicas o principalmente privadas (Ruiters y Matji, 2015, p. 672). En este escenario, las nociones de responsabilidad mutua, accesibilidad financiera, buena gobernanza, arbitraje como un sistema legal internacional, manejo integrado del agua, preservación del recurso y gobernanza global del agua toman relevancia (Schneier-Madanes, 2014). De modo que para construir un nuevo modelo de gobernanza del agua, los tres pilares consistirán en manejo integrado, recuperación de costo y la participación ciudadana (Schneier-Madanes, 2014).

Por tanto, los escenarios de la gobernanza global se constituyen a partir de instituciones, organizaciones y activistas para la formulación de políticas regionales y multiniveles, por ejemplo, Unión Europea; leyes internacionales y mecanismos de cooperación internacional, por ejemplo, convenciones, declaraciones, pactos, acuerdos interestatales, partenariados públicos-públicos, tratados internacionales, etc. (Valdovinos, 2011). Igualmente, se considerarían agencias como la ONU, responsables de la formulación de políticas globales con un enfoque de tratado y agencias centralizadas o para la formulación de políticas regionales centradas en el Estado. Tienen lugar plataformas como el Foro Mundial por el Agua como una aproximación informal, descentralizada y de mercado para la formulación de políticas globales, organizaciones globales de profesionales, organizaciones regionales enfocadas al manejo hídrico y compañías privadas de agua (Darling, 2012; PahlWostl, Gupta, y Petry, 2008b). 
Este tipo de gobernanza sería catalogada dentro de una gobernanza de tipo mobius web, en tanto contemplan una multiplicidad y heterogeneidad de arreglos, relaciones y actores (Pahl-Wostl, Gupta, y Petry, 2008b), ya que en la creación de la visión de una gobernanza global del agua, han intervenido un conjunto con gobiernos, actores privados, prestamistas, y medios de comunicación a nivel internacional, con influencias científicas, políticas y económicas (Scheneier-Madanes, 2014).

\section{CONCLUSIONES}

A pesar de la multiplicidad de enfoques, definiciones y cuestionamientos, se puede observar que la gobernanza del agua involucra aspectos como: el manejo burocrático, sistemático o institucional; dentro de la gobernabilidad, los procesos, sistemas o acciones colectivas; que requiere de la participación ciudadana, control social, rendición de cuentas, distribución de la responsabilidad, reglas formales e informales, confianza, actores, interacciones (creencias, valores, discursos, prácticas), niveles de acción, negociación de intereses para la toma de decisiones y ejercicio de poder sobre cómo administrar y conservar el agua.

Los actores pueden ser de distinta índole, heterogéneos, numerosos, públicos o privados y los niveles de acción a analizar suelen ser la cuenca, el local, regional, provincial, nacional, internacional, transnacional, y global, ante los cuales no hay un consenso definitivo sobre en qué nivel abordar la gobernanza, porque cada uno expone cierta favorabilidad, pero también plantean críticas. Habría entonces que buscar la cooperación entre los diversos actores para sostener el recurso hídrico a pesar de sus retos de representatividad, legitimidad y oposiciones. Varios de los casos expuestos evidencian que una opción favorable sería redirigir la atención de los actores estatales a las comunidades, líderes sociales y usuarios. En todo caso, implica comprender la gobernanza como acción pública en la que distintos niveles de acción indican una territorialización del agua, que se debe considerar en su complementariedad, para ejercer democracia y buscar la eficiencia administrativa.

Hay enfoques por disciplinas y por ende, objetos de estudio particulares, pero también enfoques estructurales, constructivistas, etc. que incidirá también en la manera de categorizar u operacionalizar el concepto de gobernanza del agua. Sin embargo, algunas categorías comunes que se consideran relevantes para su análisis desde una perspectiva de la democracia participativa, son los actores, relaciones entre los actores y el medio ambiente, intereses, área hídrica de interés, legislación, participación, conocimiento. Otra dimensión importante es la de poder y autoridad alrededor de la gobernanza del agua, ya sea en una perspectiva relacional o estructural entendiéndola como la capacidad de determinar decisiones e influenciar a otros actores y sobre los recursos hídricos. La gobernanza debe entonces equilibrar la centralización y descentralización, la dispersión del poder y participación social para lograr mayor inclusión en la toma de decisiones.

También hay un llamado a contextualizar las distintas formas de gobernanza, ya que éstas pueden oponerse, yuxtaponerse o mantenerse en constante tensión; debido a que dependiendo del tipo de presencia de la gobernanza y de los actores involucrados, así mismo será el alcance y complejidad de los hallazgos y análisis. Es 
por eso que hay varios tipos de gobernanza: participativa, co-gobernanza, global, local, regional, multinivel, colaborativa, tipo mobius web. Pero para lograr el consenso se requiere ejercer un componente educativo y de construcción de conocimiento.

No obstante, la propuesta principal para analizar la gobernanza es desde la multidisciplinariedad o interdisciplinariedad que a su vez requiere de un verdadero vínculo entre las diversas disciplinas, previa búsqueda de una coherencia teórica. Sin embargo, se requiere considerar la crítica para redireccionar los planteamientos y cuestionar lo que se da por sentado. Al tiempo que se le exige a los investigadores de este tipo de objetos de estudio, estar involucrados en las necesidades de la sociedad y el medio ambiente.

\section{REFERENCIAS}

1. Araral, E. y Wangb, Y. (2015). Does water governance matter to water sector performance? Evidence from ten provinces in China. Water Policy, 17, pp. 268-282.

2. Asthana, V. (2013). Transnational Policy Networks in Global Water Governance in India. Journal of International and Global Studies 5(1), pp. 61 . 79.

3. Baños, J. (2006). Teorías de la democracia: Debates actuales. Revista Andamios, 2(4), pp. 35-58.

4. Barlow, M. \& Clarke, T. (2001). Blue Gold: The Fight to Stop the Corporate Theft of the World's Water. New York: The New Press.

5. Benhamt, C., Hussey, K. y Beavis, S. (2014). Planning for success in a climate change future: Collaborative water governance in the Upper $\mathrm{Mu}$ rrumbidgee Catchment, southeastern Australia. Australian Journal of Water Resources 18 (1), pp. 1-14.

6. Boakye, M. K. \& Akpor, O. B. (2012). Stakeholder's participation in Water Management: A case study of the Msunduzi Catchment Management Forum of KwaZulu-Natal, South Africa. Journal of sustainable Development. pp. 104-112.

7. Borda, O. F. (1991). La accidentada marcha hacía la democracia participativa en Colombia. Análisis Político, pp. 46-59.

8. Brenner, L. y de La Vega Leiner, A. (2014). La gobernanza participativa de áreas naturales protegidas. El caso de la Reserva de la Biosfera El Vizcaíno. Región y Sociedad, 36 (59), pp. 183-213. 
9. Brondizio, E., Ostrom, E., \& Young, O. R. (2009). Connectivity and the governance of multinivel social-ecological systems: The role of social capital. Annual Review of environment and resources (34), pp. 253-278.

10. Cohen, M.A. (2013). Democracia deliberativa y gobernanza ambiental:iconceptos transversales de una nueva democracia ecológica? Sociológica, pp. 73-122.

11. Colmenares, R. (2013). ¿Gobernanza del agua o justicia del agua? En Seminario virtual de caja de herramientas. Edición $\mathrm{N}^{\circ} 00342$-Semana del 8 al 14 de marzo de 2013. Recuperado de http://viva.org.co/cajavirtual/ svc0342/articulo12.html.

12. Dahl, R. (2003). iEs democratica la Constitución de los Estados Unidos? Buenos Aires: Fondo de Cultura Económica.

13. Dahl, R. (1992). La democracia y sus críticos. Barcelona, Paidós.

14. Daniel, J., Pinel, S., Brooks, J. (2013). Overcoming barriers to collaborative transboundary water governance: Idetifying local strategies in a fragmented governance setting in the United States. Mountain Research and Development, 33 (3), pp. 215-224.

15. Darling, K. (2012). A weight for water: an ecological feminist critique of emerging norms and trends in global water governance. Melbourne Journal of International Law, 13, pp. 368-394.

16. Domínguez Serrano, J. (2010). El acceso al agua y saneamiento: Un problema de capacidad institucional local Análisis en el estado de Veracruz. Gestión y Política Pública, 19(2), pp. 311-350.

17. Fracalanza, A. P., Martins Jacob, A. y Furtado Eça, R. (2013). Justicia Ambiental y Prácticas de gobernanza del agua: (Re) Introduciendo cuestiones de igualdad en la agenda. Revista Ambiente e Sociedade, XVI (1), pp. 19-38.

18. Goodwin, B. (1997). El uso de las ideas políticas. Ed. Península.

19. Grecksch, K. (2015). Adaptive capacity and water governance in the Keiskamma River Catchment, Eastern Cape Province, South Africa. Water SA, 41(3), pp. 359-368.

20. Held, D. (1997). La Democracia y el Orden Global. (S. Mazzuca, Trad.) Barcelona: Paidós Ibérica.

21. Hevia, F. (2011). Participación ciudadana institucionalizada y organizaciones civiles en Brasil: articulaciones horizontales y verticales en la política de asistencia social. Revista de Estudios Sociales, Universidad de los Andes Bogotá, 39, pp. 95-108. 
22. Hill, C., Furlong, K., Bakker, K., y Cohen, A. (2008). Harmonization vs. subsidiarity in wáter governance: a review of wáter governance and legislation in the Canadian provinces and territories. Canadian Water Resources Journal, 33(4), pp. 315-332.

23. Horne, A. y O'Donnell, E. (2014). Decision making roles and responsibility for environmental water in the Murray-Darling Basin. Australian Journal of Water Resources, 18 (2), pp. 118-132.

24. Howard, J. (2006). Using game theory to explain the behaviour of participants involved in a regional governance process. Rural Society 16 (3), 254-270.

25. Landázuri, G. (2008). Desarrollo y participación. En Gatica, I. et al. (coords.). Poder, actores e instituciones. Enfoques para su análisis. México: Eón-UAM.

26. Lemos, M. C. \& Agrawal, A. (2006). Environmental governance. Annual Review of environment and reosurces (31), pp. 297-325.

27. Lindblom, C. E. (1991). El proceso de elaboración de políticas públicas. Madrid: Ministerio para las administraciones públicas.

28. Marín Aranguren, M. (2010). La sociedad civil global en la gobernanza ambiental del sector agua en el mundo. OASIS-Observatorio de análisis de los sistemas internacionales, 15, pp. 53-73.

29. Marti, J. L. (2006). La republica deliberativa. Una teoría de la democracia. Madrid: Marcial Pons.

30. Meissner, R. (2014). Who wants to be an agent? A framework to analyse water politics and governance, Water SA, 40 (1), pp. 1-9.

31. Mejía Quintana, O. y Blanco Blanco, J. (2005). Democracia y Filosofía de la Historia en América Latina. Bogotá: Ediciones Jurídicas Gustavo Ibañez Ltda.

32. Motta V. R. (2010). El derecho humano al agua potable: entre un reconocimiento popular y jurisprudencial. Revista de Derecho y Ciencias Sociales, 3, pp. 257-272.

33. Motta V.R. (2013). Gobernanza del agua en Cundinamarca para garantizar un derecho. Revista Republicana, 15, pp. 163-180.

34. Mumme, S., Ibáñez, O. y Till, S. (2012). Multilevel governance of wáter on the US-Mexico border. Regions \& Cohesion, 2 (2), pp. 6-29.

35. Oszlak, O. (2009). Implementación participativa de las políticas públicas: Aportes a la construcción de un marco analítico. Buenos Aires: Cippec, Presidencia de la Nación. 
36. Paerregaard, K. (2013). Governing wáter in the Andean Community of Cabanaconde, Peru. Mountain research and Development, 33 (3), pp. 207. 2014.

37. Pahl-Wostl, C., Gupta, J. y Petry, D. (2008). Governance and the Global Water System: A Theoretical Exploration. Global Governance, 14, pp. 419-435.

38. Peters, G. (2007). Globalización, gobernanza y estado: Algunas proposiciones acerca del proceso de gobernar. Revista CLAD Reforma y Democracia, 39 .

39. Ruano, A. L. (2011). 'If no one else stands up, you have to': a story of community participation and water in rural Guatemala. Global Healt Action (4), pp. 1-10.

40. Ruiters, C. y Matji, M. (2015). Water institutions and governance models for the funding, financing and management of water infrastructure in South Africa. Water SA, 41(5), pp. 660-676.

41. Ruiz, S. y Gentes, I. (2008). Retos y perspectivas de la gobernanza del agua y gestion integral de recursos hídricos en Bolivia. European Review of Latin American and Caribbean Studies, 85, pp. 41-59.

42. Sandoval, D. M. (2013). Protected areas in the city, Urban wetlands of Bogotá. Cuadernos de vivienda y urbanismo, 11(6), pp. 80-103.

43. Sartori, G. (1994). ¿Qué es la democracia? Bogotá: Altamir Editores.

44. Schneier-Madanes, G. (2014). Introduction. En Graciela Schneier-Madanes (ed.), Globalized Water. A Question of Governance, (pp. 1-15). Doi: 10.1007/978-94-007-7323-3.

45. Sentencia, T 406 (Corte Constitucional 1992).

46. Sorj, B. (2005). La democracia inesperada. Buenos Aires: Universidad de Bologna.

47. Ünver, O. (2008). Global Governance of Water: A Practitioner's Perspective. Global Governance, 14, pp. 409-417.

48. Uribe, M. T. (2002). Las promesas incumplidas de la democracia participativa. En A. Varios, Debate a la Constitución. Bogotá: Instituto latinoamericano de Servicios Legales Alterntivos.

49. Valdovinos, J. (2011). De la gestión local a la gobernanza global. Actores e interacciones multiniveles en la gestión del agua en la ciudad de México. Regions \& Cohesion, 1(3), pp. 34-66.

50. Veiga, D. (2009). Desigualdades sociales y fragmentaciVeigaón urbana. En Poggiese, H., Cohen Egler, T. (comp.). Otro Desarrollo Urbano: ciudad incluyente, justicia social y gestión democrática (pp. 51-61). Buenos Aires: CLACSO. 
LA DEMOCRACIA PARTICIPATIVA COMO PRINCIPIO CONSTITUCIONAL: ALGUNOS APORTES PARA LA Gobernanza del agua en COlombia

51. Xiang, L.(2015). Systematic methodology and practice of a co-governance program for five waters in a typical area of China, Water Policy, 17, pp. 804-814.

52. Zhou, S., Du, A. y Bai, M. (2015). Application of the environmental Gini coefficient in allocating water governance responsibilities: a case study in Taihu Lake Basin, China. Water science \& Technology, 71(7), pp. 10471055 .

* Lyda Teresa Córdoba Hoyos

Magíster en Políticas Públicas.

Politóloga y Candidata a Doctora en Administración.

Investigadora del grupo "Derecho, Sociedad y Estado - GIDSE", Universidad del Valle, Cali, Colombia.

Docente, Facultad de Ciencias de la Administración y del Instituto de Estudios Políticos y Sociales, Universidad del Valle, Cali, Colombia.

ORCID: https://orcid.org/0000-0002-1792-0693

Correo electrónico: lyda.cordoba@correounivalle.edu.co

**Jorge Enrique Arias Calderón

Doctor en Derecho Constitucional.

Profesor Asociado, Facultad de Ciencias de la Administración, Universidad del Valle, Cali, Colombia.

Director del Grupo de investigación "Derecho, Sociedad y Estado GIDSE", Universidad del Valle, Cali, Colombia.

ORCID: https://orcid.org/0000-0001-8602-7068

Correo electrónico:jorge.arias@correounivalle.edu.co 